\title{
Optical gain in carbon nanotubes
}

\author{
Etienne Gaufrès, ${ }^{1}$ Nicolas Izard,,${ }^{1,2}$ Xavier Le Roux, ${ }^{1}$ Delphine \\ Marris-Morini, ${ }^{1}$ Saïd Kazaoui, ${ }^{2}$ Eric Cassan, ${ }^{1}$ and Laurent Vivien ${ }^{1}$ \\ ${ }^{1}$ Institut d'Electronique Fondamentale, CNRS-UMR 8622, Université Paris-Sud 11, 91405 Orsay, France \\ ${ }^{2}$ National Institute of Advance Industrial Science and Technology (AIST), Tsukuba, Japan
}

(Dated: Received June 15, 2022)

\begin{abstract}
Semiconducting single wall carbon nanotubes (s-SWNT) have proved to be promising material for nanophotonics and optoelectronics. Due to the possibility of tuning their direct band gap and controlling excitonic recombinations in the near-infrared wavelength range, s-SWNT can be used as efficient light emitters. We report the first experimental demonstration of room temperature intrinsic optical gain as high as $190 \mathrm{~cm}^{-1}$ at a wavelength of $1.3 \mu \mathrm{m}$ in a thin film doped with $(8,7)$ s-SWNT. These results constitute a significant milestone towards the development of laser sources based on carbon nanotubes for future high performance integrated circuits.
\end{abstract}

PACS numbers: 42.55.-f, 78.45.+h, 78.55.-m, 78.67.Ch, 81.07.De

Unique electronic[1, 2] and optical[3] properties of Single-Wall Carbon Nanotubes (SWNT) allow them to play a major role in futur photonic circuits 4,6 . Since the discovery of photoluminescence of nanotubes encapsulated into micelle surfactant [7, research efforts in nanotube photonics have greatly intensified, and several building blocks have already been demonstrated, such as field-effect transistor [1] or detectors 4. Luminescence properties of carbon nanotubes attracted a special interest, and became a leading component in various configurations, not only as individualised entities in micelle surfactant, but also when suspended over trenches $[8$ or deposited onto chip surface 9 . Numerous works prove that the nanotube environment plays a primordial role in their emission behaviour 10 12, and several nonradiative de-excitation mechanisms exist, either by Auger recombination 13 or energy transfers to metallic SWNT (m-SWNT) [14 or excitons 15]. These mechanisms lead to a strong competition between bright and dark excitons and a limited photoluminescence lifetime [16. Up to now, a main challenge was to obtain light amplification in carbon nanotubes. Here, we experimentally demonstrate optical gain in carbon nanotubes at a wavelength of $1.3 \mu \mathrm{m}$ at room temperature.

Semiconducting SWNT (s-SWNT) extracted by the previously described polyfluorene (PFO) method 17. were drop cast on glass substrate. Pure s-SWNT thin films will be hereafter designated as sample A, while a control sample made with a blend of s- and m-SWNT will be named sample B. Both layers have a homogeneous carbon nanotube density and a thickness of $1 \mu \mathrm{m}$. The coating was made to obtain regular and vertical edge facets[18. Absorbance spectrum of such initial sSWNT/PFO solution and photoluminescence spectrum of drop-casted s-SWNT/PFO solution on glass substrate are reported in ref 12, Optical properties characterized by the $E_{22}$ and $E_{11}$ optical transitions are related to strongly bound excitons 19 . Photons absorbed on the $\mathrm{E}_{22}$ optical transition create excitons at the carbon nanotube surface and light emission is produced due to the exciton recombination through the $\mathrm{E}_{11}$ optical transi-

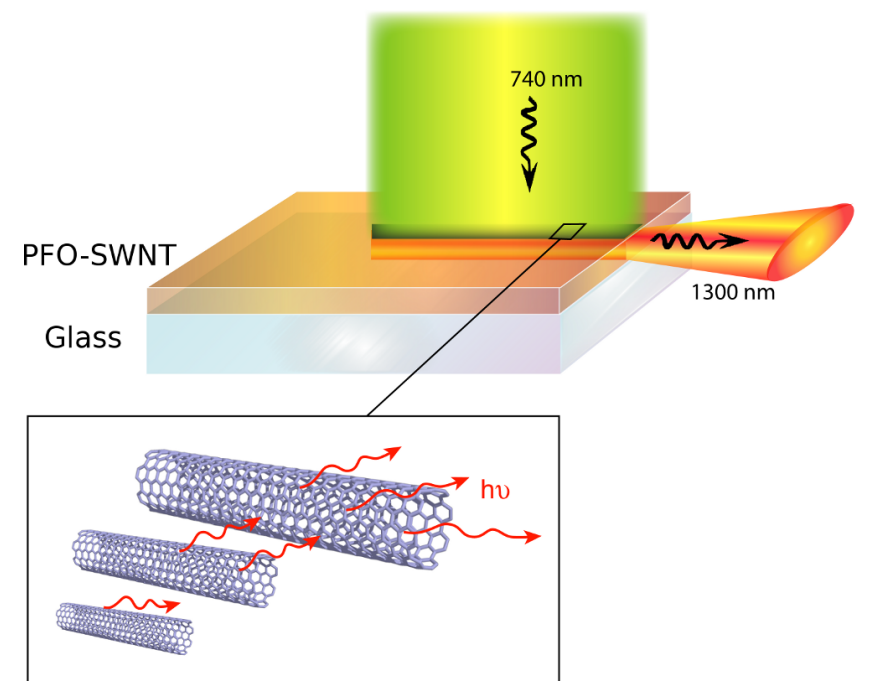

FIG. 1. (Color online) Schematic representation of a Variable Scan Length (VSL) experiment.

tion. The polymer host matrix (PFO) presents absorption and photoluminescence peaks in the UV-visible spectrum range, and is optically inactive (no absorption and no emission) on the near infrared carbon nanotube absorption and emission range, without any evidence of energy transfer towards carbon nanotubes 20].

Optical gain measurements were first performed on sample A using the variable strip length (VSL) method 21]. The sample was optically excited by an Optical Parametric Oscillator laser emitting at a wavelength of $740 \mathrm{~nm}$, pumped by a two nanosecond pulsed Nd:YAG laser at $355 \mathrm{~nm}$. Amplified Spontaneous Emission (ASE) of carbon nanotubes was then collected at the edge of the sample as a function of the excitation length $l$, as schematically described in Fig. 1. Fig. 2(b) presents the ASE intensity $\left(I_{A S E}\right)$ of sample A as a function of length $l$, for low and high pump fluences $\left(35 \mathrm{~mJ} \cdot \mathrm{cm}^{-2}\right.$ and $500 \mathrm{~mJ} \cdot \mathrm{cm}^{-2}$, respectively). At high fluence, a strong 
signal increase was observed, while at low fluence, saturation of the luminescence signal was directly reached. The difference in the ASE responses under low and high excitations demonstrated a clear change of optical behaviour which clearly proved optical gain feature in sample A 22, 23. $I_{A S E}$ can be described by the one dimensional amplifier model[22]:

$$
I_{A S E}(l) \sim \frac{I_{\text {Spont }}}{g_{\text {int }}-\alpha} \cdot\left(e^{\left(g_{\text {int }}-\alpha\right) \cdot l}-1\right)
$$

were perfectly fitted as shown in Fig.1C, where the theoretical curves for high and low pump fluences are respectively drawn in red and blue solid lines. The ASE intensity evolution featured an exponential increase at high pump fluence, while an exponential saturation at low pump fluence was obtained. The net modal gain $g_{\text {net }}$ at high fluence was estimated to be about $160 \pm 10 \mathrm{~cm}^{-1}$. This gain value for carbon nanotubes embedded in polymer films was of the same order of magnitude than the gain measured in other reference semiconducting nanomaterial systems, such as silicon nanocrystals 23. For long strip length excitation ( $l>0.25 \mathrm{~mm})$ and high fluence, a saturation of $I_{A S E}$ was observed. Main origins of this saturation were a geometrical factor caused by the reduction of solid angle emission and the increased absorption length by the nanotube doped layer, and a gain saturation effect caused by the depletion of the excited state population under high ASE intensity 24. At low fluence, the intrinsic gain $g_{\text {int }}$ of SWNT was too low to compensate for loss, and the measured net modal gain $g_{\text {net }}$ was then negative. Using equation 1, a fit to experimental data gave an estimation of the net modal gain $g_{\text {net }}$ of about $-32 \mathrm{~cm}^{-1}$. The same VSL experiments were also performed on sample $\mathrm{B}$, which contained both semiconducting and metallic carbon nanotube. VSL experiments were performed at pump fluences from 90 to $590 \mathrm{~mJ} \cdot \mathrm{cm}^{-2}$, and the results are reported in Fig. 2(b). Exponential saturations were observed for each input pump fluence which, according to equation 1, corresponded to negative net modal gain. For pump fluences from 90 to $590 \mathrm{~mJ} \cdot \mathrm{cm}^{-2}$, the net gain varied from $-41 \mathrm{~cm}^{-1}$ to $-19 \mathrm{~cm}^{-1}$. This underlines the importance of the absence of $\mathrm{m}-\mathrm{SWNT}$ and nanoparticles in a sample on the optical gain properties: indeed, sample A was only constituted by s-SWNT in PFO 12, 17.

In order to provide an estimation of the overall optical losses $\alpha$, the Shifting Excitation Spot (SES) method was used 24. Furthermore, this method allows a scan of the VSL strip length with a constant size excitation spot and is able to demonstrate the homogeneity of the thin SWNT based layer. SES experiments were performed on samples A and B, and results are reported in Fig. 3(a). First, $I_{A S E}$ increased and quickly reached a maximum when the spot no more interacted with the sample edge. After this peak, a monotone decrease was observed in log scale for both samples, as the distance between the spot position and the sample edge increased. First, the monotone decrease confirmed the good optical homogeneity of
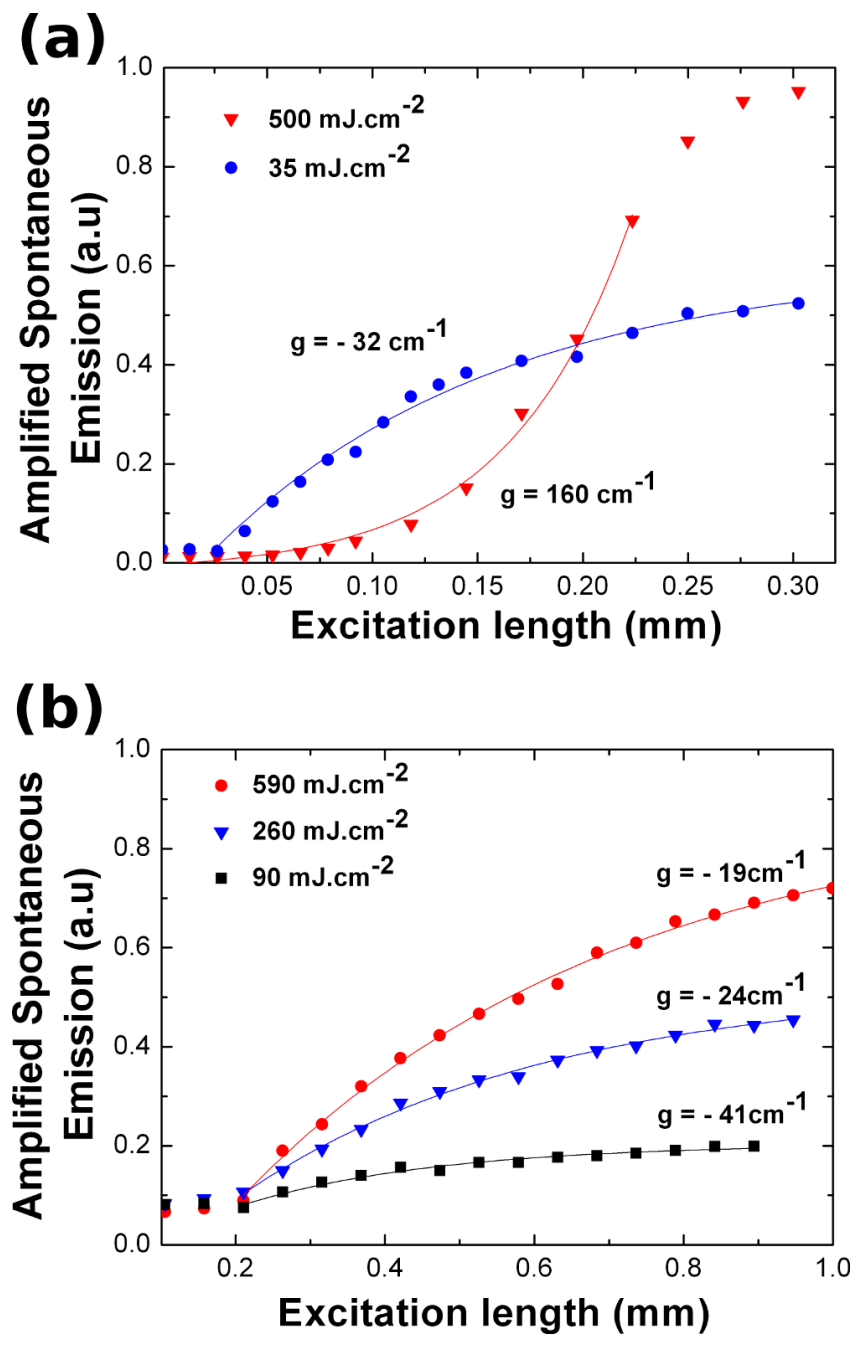

FIG. 2. (Color online) (a) $I_{A S E}$ vs excitation stripe length $l$ of pure s-SWNT embedded in thin layer (Sample A). Blue and red dots correspond to two input fluences of the excitation: $35 \mathrm{~mJ} \cdot \mathrm{cm}^{-2}$ and $500 \mathrm{~mJ} \cdot \mathrm{cm}^{-2}$, respectively. Excitation and recording wavelengths were respectively $740 \mathrm{~nm}$ and $1300 \mathrm{~nm}$. A fit to the data with equation 1 is shown as a solid line. For sample A, $g_{\text {net }}$ was $140 \mathrm{~cm}^{-1}$ in high fluence regime and $-19 \mathrm{~cm}^{-1}$ at low fluence regime. (b) $I_{A S E}$ vs excitation stripe length $l$ of SWNT embedded in thin layer composed of a mixture of s- and m-SWNT (Sample B). The input pump fluences were explored under the same experimental condition as in Fig. 2(a).

both samples, and the evolution of $I_{A S E}$ is described by the following relation $[22]$ :

$$
I_{A S E}(x) \sim I_{\text {Spont }} \cdot e^{-\alpha \cdot x}
$$

where $I_{\text {Spont }}$ is the spontaneous emission intensity per length unit and $x$ the distance between the excitation spot and the sample edge. According to equation 2 and SES measurements, loss coefficients of both samples A and $\mathrm{B}$ were $37 \mathrm{~cm}^{-1}$ and $50 \mathrm{~cm}^{-1}$, respectively. Optical losses in sample B were much higher than in sample A, which was directly related to the presence of $\mathrm{m}-\mathrm{SWNT}$ 

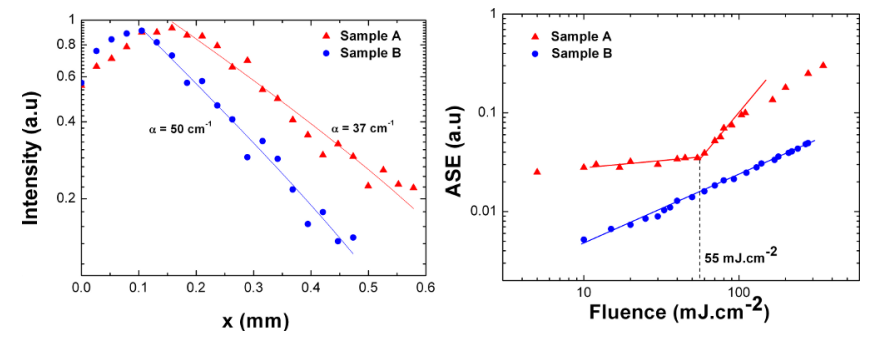

FIG. 3. (Color online) (a) SES intensity as a function of the excitation spot position $x$ for sample A and B. A fit with equation 2 gave the overall losses in the thin layer composite. (b) Edge-emission intensity of the $(8,7) \mathrm{s}-\mathrm{SWNT}$ at $1300 \mathrm{~nm}$ as a function of pump fluence at room temperature for both samples A and B. A significant gain energy threshold was observed around $55 \mathrm{~mJ} \cdot \mathrm{cm}^{-2}$ for sample A. Solid lines are guide for the eye.

and nano-particles, and increasing light absorption. This increase of loss and the less efficient light emission obtained in sample B can explain why a positive net modal gain could not be observed. However, from the determination of the optical loss $\alpha$ using the SES method and the net modal gain $g_{\text {net }}$ obtained from the VSL method, the intrinsic gain $g_{\text {int }}=g_{\text {net }}+\alpha$ for sample A was as high as $190 \pm 10 \mathrm{~cm}^{-1}$.

The evolution of $I_{A S E}$ as a function of the incident laser fluence was also studied (Fig. 2(b)). Experimental conditions were identical for both samples A and B. In the case of sample A, a threshold pump fluence was observed around $55 \mathrm{~mJ} \cdot \mathrm{cm}^{-2}$. Above the threshold, ASE intensity presented a significant increase followed by a slight saturation. This is a typical signature of light amplification due to an optical gain. Below the pump fluence threshold, light emission was dominated by the losses from the absorption peaks, while above the fluence threshold, the optical gain dominated the losses, resulting in an enhancement of light emission. On the contrary, such a behaviour was not observed with sample B for which the ASE intensity behaviour was only driven by the optical loss.

Normalized photoluminescence spectra of nanotube in sample A for two input pump fluences are reported in Fig. 4. Both fluences were chosen to be below and above the fluence threshold as determined in Fig. 3(b). A linewidth narrowing of $29 \%$ was observed for the $(8,7)$ nanotube at $1300 \mathrm{~nm}$. This result also constituted a clear demonstration of optical gain in sample A 25. No linewidth narrowing was observed for sample B. We could also observe a similar linewidth narrowing feature in $(8,6)$ nanotube at $1200 \mathrm{~nm}$, providing evidence for optical gain at several wavelengths.

These experimental results clearly indicate significant optical gain in pure semiconducting carbon nanotubes embedded in host polymer thin film. A few metallic carbon nanotubes mixed with s-SWNT can strongly affect the emission behavior and, as observed in sample B, prohibit the detection of optical gain. The ability to generate

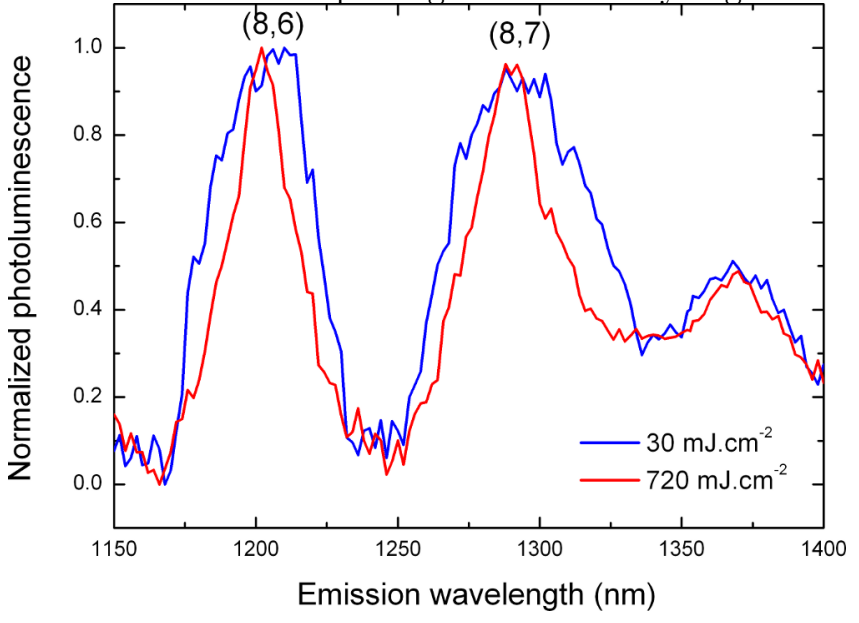

FIG. 4. (Color online) High resolution normalized spectra of the ASE for sample A at low $\left(30 \mathrm{~mJ} \cdot \mathrm{cm}^{-2}\right)$ and high $\left(720 \mathrm{~mJ} \cdot \mathrm{cm}^{-2}\right)$ pump fluences with an excitation wavelength of $740 \mathrm{~nm}$. A $29 \%$ linewidth narrowing (FWHM from 63 to $45 \mathrm{~nm})$ was observed on the photoluminescence of $(8,7)$ nanotube at $1300 \mathrm{~nm}$, and linewidth narrowing of $28 \%$ (FWHM from 44 to $32 \mathrm{~nm}$ ) was also observed for the $(8,6)$ nanotube at $1200 \mathrm{~nm}$. Both spectra intensity were normalized to 1 , considering the signal around $1170 \mathrm{~nm}$ as zero[18]

and detect and intrinsic gain of $190 \mathrm{~cm}^{-1}$ in carbon nanotubes is the first step toward the development of carbon nanotubes-based laser and more generally toward photonic circuits based on carbon nanotubes.

\section{ACKNOWLEDGMENTS}

We thank R. Colombelli and S. Laval for insights and fruitful discussion, A. Charrier for her help with graphic arts, D. Riehl from DGA for his help with beam profile measurements and C.T. Cheung for her thoroughly proofreading of the manuscript. N.Izard thanks the Japan Society for Promotion of Science and CNRS for financial support.
[1] R. Martel, V. Derycke, C. Lavoie, J. Appenzeller, K.K. Chan, J.Tersoff, Ph. Avouris, Phys. Rev. Lett., 87, 256805 (2001)
[2] H. Postma, T. Teepen, Z. Yao, M. Grifoni, C. Dekker, Science, 293, 76 (2001)

[3] Ph. Avouris, J. Chen, Materials Today, 9, 46 (2006) 
[4] M. Itkis, F. Borondics, A. Yu, R. Haddon, Science, 312, 413 (2006)

[5] J.A. Misewich, R. Martel, Ph. Avouris, J.C. Tsang, S. Heinze, J. Tersoff, Science, 300, 783 (2003)

[6] F. Xia, M. Steiner, Y.-M. Li, Ph. Avouris, Nature Nanotechnology, 3, 609 (2008)

[7] S.M. Bachilo, M.S. Strano, C. Kittrell, R.H. Hauge, R.E. Smalley, R.B. Weisman, Science, 298, 2361 (2002)

[8] J. Lefebvre, D. Austing, J. Bond, P. Finnie, Nano Letters, 6, 1603 (2006)

[9] E. Adam, C. M. Aguirre, L. Marty, B.C. St-Antoine, F. Meunier, P. Desjardins, D. Mnard, R. Martel, Nano Letters, 8, 2351 (2008)

[10] P. Finnie, Y. Homma, J. Lefebvre, Phys. Rev. Lett., 94, 247401 (2005)

[11] T. Okazaki, T. Saito, K. Matsuura, S. Ohshima, M. Yumura, S. Iijima, Nano Letters, 5, 2618 (2005)

[12] E. Gaufrès, N. Izard, L. Vivien, S. Kazaoui, D. MarrisMorini, E. Cassan, Opt. Lett., 34, 3845 (2009)

[13] F. Wang, G. Dukovic, E. Knoesel, L. Brus, T. Heinz, Phys. Rev. B, 70, 241403 (2004)

[14] T. Hertel, R. Fasel, G. Moos, Appl. Phys. A, 75, 449 (2002)

[15] I. Mortimer, R. Nicholas, Phys. Rev. Lett., 98, 027404 (2007)
[16] F. Wang, G. Dukovic, L. Brus, T. Heinz, Phys. Rev. Lett., 92, 177401 (2004)

[17] N. Izard, S. Kazaoui, K. Hata, T. Okazaki, T. Saito, S. Iijima, N. Minami, Appl. Phys. Lett., 92, 243112 (2008)

[18] See supplementary material at [URL] for experimental details, film roughness measurements and uncorrected photoluminescence spectra.

[19] F. Wang, G. Dukovic, L. Brus, T. Heinz, Science, 308, 838 (2005)

[20] A. Nish, J.Y. Hwang, J. Doig, R. Nicholas, Nanotechnology, 19, 095603 (2008)

[21] K. Shaklee, R. Leheny, Appl. Phys. Lett., 18, 475 (1971)

[22] K. Shaklee, R. Nahory, R. Leheny, Journal of Luminescence, 7, 284 (1973)

[23] L. Pavesi, L.D. Negro, C. Mazzoleni, G. Franzo, F. Priolo, Nature, 408, 440 (2000)

[24] L.D. Negro, P. Bettotti, M. Cazzanelli, D. Pacifici, L. Pavesi, Optics Communications, 229, 337 (2004)

[25] T. Quist, R.H. Rediker, R.J. Keyes, W.E. Krag, B. Lax, A.L. McWhorter, H.J. Zeigler, Appl. Phys. Lett., 1, 91 (1962) 